no contact, or if the indifferent lead is broken, the circuit may still be completed by leakage of the current to earth, particularly if the machine is producing frequencies above $1 \mathrm{MHz}$. The earth lead of the power supply cable forms the final circuit link to the diathermy machine. If any part of the patient's body touches an earthed metal fitting such as a drip stand or arm rest, leakage occurs preferentially along this path and a burn develops at the point of contact.

New types of diathermy machines are now available, some with transistorised circuits which may incorporate additional safety features. In some new machines the diathermy circuit is isolated from earth. If the indifferent electrode is not applied to the patient, or if its lead breaks, the circuit cannot be completed by current leakage to earth, and hence the machine should fail to function. The newly available machines have been tested in Bristol, ${ }^{3}$ including one machine that is claimed to incorporate an earth-free diathermy circuit. Provided the intensity of the current was increased, this worked satisfactorily, even when the indifferent electrode was suspended in mid-air, out of contact with any object. This, and other machines that functioned in this manner, operated at frequencies above $1.5 \mathrm{MHz}$. It was thought that at high frequencies stray leakage of current might be as much as $25 \%$. Some machines operated satisfactorily when the indifferent electrode was placed on the top of the diathermy cabinet, or on the pedestal of the operating table, or connected to an earthed object such as the anaesthetist's trolley or theatre radiator pipes.

The surgeon, as well as the patient, may be at risk from burns from conventional diathermy machines, particularly when using endoscopes. ${ }^{4}$ Earth-free diathermy circuits are claimed to protect the surgeon from periocular burns during endoscopic diathermy procedures, but this was found not to be so when using machines operating above $1.5 \mathrm{MHz}$. Manufacturers should be encouraged to produce machines with frequencies below $1 \mathrm{MHz}$ and to provide insulated covers for the diathermy cabinets. Even more important, surgeons and theatre staff should remain cautious when using any diathermy machine, and should not consider it foolproof.

${ }^{1}$ Briscoe, C E, Hill, D W, and Payne, J P, British fournal of Surgery, 1976, 63, 981.

2 Mitchell, J P, Lumb, G N, and Dobbie, A K, A Handbook of Surgical Diathermy, 2nd edn. Bristol, John Wright, 1978.

${ }^{3}$ Mitchell, J P, Annals of the Royal College of Surgeons of England, 1979, 61, 287.

4 Mitchell, J P, and Lumb, G N, Proceedings of the Royal Society of Medicine, 1960, 53, 348.

\section{Benign recurrent vertigo}

Patients often complain of dizziness and disorders of equilibrium, but their symptoms need careful and precise analysis to determine whether they do indeed suffer from vertigo or from light-headedness or some other complaint. Vertigobest defined as an illusion of altered position or motion that may have objective manifestations-may be the presenting symptom of many different diseases, including temporal-lobe epilepsy, acute ophthalmoplegia, conditions associated with raised intracranial pressure, and some cerebellar disorders; vascular, post-traumatic, congenital, neoplastic, infectious or demyelinating processes affecting the brain stem; cerebello- pontine-angle lesions and lesions of the upper cervical roots; abnormal reactions to a number of drugs; and systemic conditions such as anaemia or hepatic dysfunction. Nevertheless, despite the large number of illnesses in which vertigo may play a part, the most common cause is disordered function of the labyrinth, and the condition most often responsible is Menière's disease.

Diagnosis requires, then, a careful clinical history with physical and neurological examination, particular attention being paid to eye movements. Simple tests of positional nystagmus and other bedside tests of eighth nerve function should be included. Caloric testing, audiometry, radiographs of the skull and internal auditory meatuses, electronystagmography, and an electroencephalogram make up the routine investigations.

At the end of all these tests certain cases of doubtful vertigo without cochlear signs remain a diagnostic problem. Some of the patients may be suffering from benign recurrent vertigo, a term used by Slater ${ }^{1}$ to describe a syndrome of repeated attacks of acute vertigo, which last from minutes to hours and are followed by a period in which positional nystagmus may be found if looked for. The attacks vary in frequency from once a day to once or twice a year. All findings are entirely normal except for the results of electronystagmography, which show some labyrinthine abnormality. Among the clinical characteristics are a strong family history and precipitation of the vertiginous attacks by lack of sleep, emotional stress, or alcohol. Some patients have headaches strongly suggestive of migraine.

The aetiology of the syndrome is unknown. It bears some resemblance to benign positional vertigo ${ }^{2}$ or vestibular neuronitis, ${ }^{3}$ but there are distinct differences. In children a similar disorder has been called benign paroxysmal vertigo of childhood $^{4}$ or paroxysmal dysequilibrium of childhood. ${ }^{5}$ The disease known as familial paroxysmal nystagmus with vertigo and ataxia ${ }^{6}$ also resembles the syndrome delineated by Slater. Again there tends to be a strong family history and precipitation of attacks by alcohol and lack of sleep.

Slater considers that benign recurrent vertigo may be a variant of migraine: certainly there is good evidence that migraine may affect the vestibular apparatus, ${ }^{7}$ and there are several reports of vertigo in migraine. ${ }^{7-10}$ In such circumstances vertigo has been labelled ${ }^{11}$ a "migraine equivalent"; it may also be seen as a "disorder of an episodic type occurring in migraine patients."12 Whatever the diagnostic label, the syndrome has a good prognosis. Slater did not use drugs in the treatment of his patients with benign recurrent vertigo, but prophylactic propranolol and pizotifen have given excellent results. Recognition of the clinical syndrome is therefore important because of its benign nature and response to treatment.

\footnotetext{
1 Slater, R, Fournal of Neurology, Neurosurgery, and Psychiatry, 1979, 42, 363.

2 Dix, M R, and Hallpike, C S, Proceedings of the Royal Society of Medicine, $1952,45,341$.

${ }^{3}$ Harrison, M S, Brain, 1962, 85, 613.

${ }^{4}$ Koenigsberger, M R, et al, Neurology, 1970, 20, 1108.

5 Watson, P, and Steele, J C, Archives of Otolaryngology, 1974, 99, 177.

6 White, J C, Archives of Neurology, 1969, 20, 276.

${ }^{7}$ Dursteler, M R, fournal of Neurology, 1975, 4, 253.

${ }^{8}$ Boenheim, F, Zentralblatt für Neurochirurgie, 1917, 36, 226.

9 Loh, T G, and Chawla, J C, Singapore Medical fournal, 1972, 13, 298.

${ }^{10}$ Eadie, M J, Medical Fournal of Australia, 1968, 2, 453.

${ }_{11}$ Graham, J R, in Handbook of Clinical Neurology, vol 5, ed P J Vinken and G W Bruyn, p 45. Amsterdam, North Holland Publishing Company, 1975.

12 Bruyn, G W, in Handbook of Clinical Neurology, vol 5, ed P J Vinken and $\mathrm{G} W$ Bruyn, p 59. Amsterdam, North Holland Publishing Company,
} 1975. 